\title{
CONSEQUENCE OF INSERTION TRAUMA - effect on early measures when using intracerebral devices
}

Ted CK Andelius ${ }^{1}$, Mette V Pedersen', Nikolaj Bøgh², Camilla Omann², Vibeke E Hjortdal' ${ }^{2}$, Michael Pedersen³, Kasper J Kyng ${ }^{1}$, Tine B Henriksen'

1) Dept. of Paediatrics, 2) Dept. of Cardiothoracic and Vascular Surgery, and 3) Comparative Medicine Lab at Aarhus University Hospital, Palle Juul-Jensens Blvd. 99, 8200 Aarhus N, Denmark

\section{INTRODUCTION}

Animal models are often used for research in neonatal hypoxic ischemic encephalopathy (HIE). Intracerebral devices may be used to quantify biological processes in cerebral tissue. Installing such a device causes an insertion trauma, which may affect early measures.

Previous studies suggest minimum one hour of observation before acquiring first measures when using microdialysis. It is unknown whether this applies to other intracerebral devices.

\section{MATERIAL AND METHODS}

10 piglets $(<24$ hours old) were anaesthetized and ventilated. Three burr holes were made $3 \mathrm{~mm}$ apart and three devices were installed $10 \mathrm{~mm}$ into the left hemisphere:

- A microdialysis catheter measuring pyruvate, glucose, glycerol, and lactate

- A probe measuring flow and oxygen tension

- A probe measuring intracranial pressure

A near infrared spectroscopy (NIRS) monitor was placed on the scalp. Measures were acquired hourly for 20 hours. $95 \%$ confidence interval at 20 hours was used to determine steadystate range.

\section{AIM}

To investigate the time needed to reach steady state when measuring intracerebral metabolism, flow, and pressure in a piglet model of HIE.

\section{CONCLUSION}

Using this setup, we recommend minimum five hours of observation to obtain correct baseline values and valid comparisons between individuals when using intracerebral devices

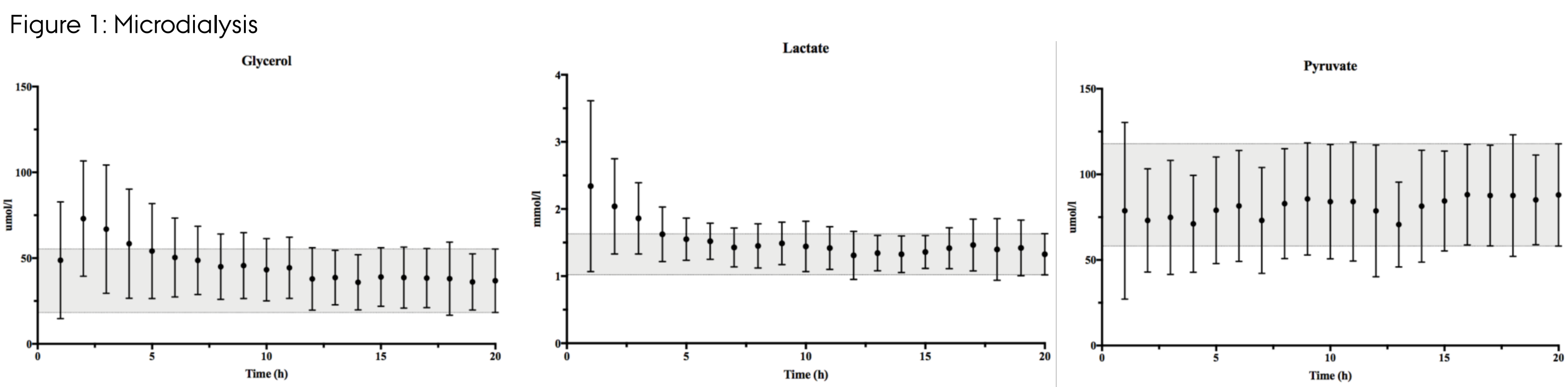

Figure 2: Probes and NIRS

oz tension
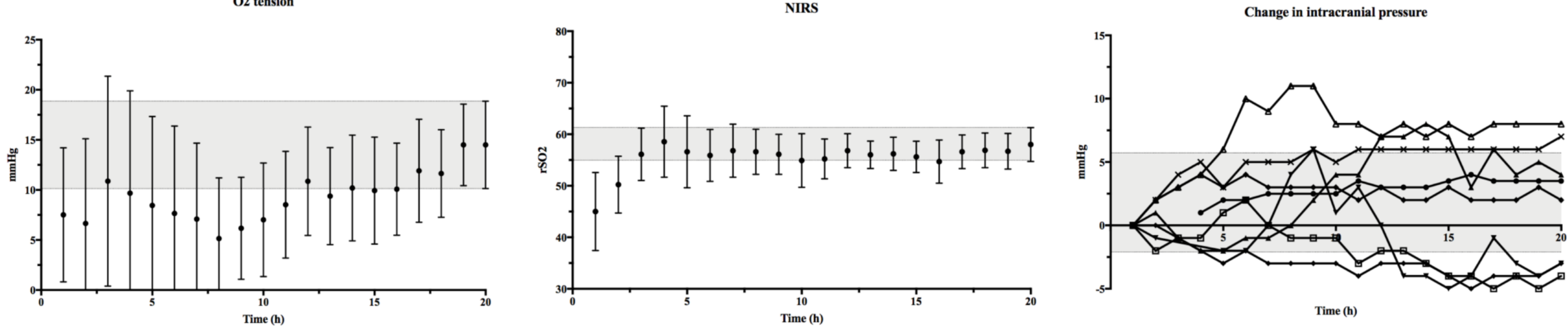

\section{RESULTS}

Steady state occurred later than expected in most measures. Glucose and pyruvate reached steady state immediately after insertion while lactate and glycerol required approximately five hours. Change in intracranial pressure reached steady state immediately, while oxygen tension and NIRS required 12 and three hours respectively. Inter-individual variability decreased for most measures until 6-7 hours. 